\title{
Téoros
}

Revue de recherche en tourisme

\section{Montréal et Québec, l'expérience touristique de morphologie nord-américaine}

\section{Danielle Pilette}

Volume 21, numéro 1, printemps 2002

Les villes capitales

URI : https://id.erudit.org/iderudit/1071536ar

DOI : https://doi.org/10.7202/1071536ar

Aller au sommaire du numéro

Éditeur(s)

Université du Québec à Montréal

ISSN

0712-8657 (imprimé)

1923-2705 (numérique)

Découvrir la revue

Citer cet article

Pilette, D. (2002). Montréal et Québec, l'expérience touristique de morphologie nord-américaine. Téoros, 21(1), 29-31. https://doi.org/10.7202/1071536ar d'utilisation que vous pouvez consulter en ligne.

https://apropos.erudit.org/fr/usagers/politique-dutilisation/ 


\section{Montréal et Québec, I'expérience touristique de morphologie nord-américaine}

\section{Danielle Pilette}

Tourisme urbain : imprécision conjuguée de deux notions évolutives

D epuis le XIX siècle, les définitions de la ville et de l'urbain sont diverses, refletant les mutations successives de l'espace et de son appropriation ainsi que la complexité du phénomène urbain. Alors que la ville traditionnelle, particulièrement européenne, sous-entend l'idée de finitude, on observe en Amérique du Nord, surtout après la Deuxième Guerre mondiale, l'émergence de nouveaux espaces urbains spécialisés, non seulement résidentiels, mais aussi industriels et d'affaires, qui permettent la restructuration sociale et économique par la ségrégation et la hiérarchisation. Qu'il s'agisse de métropole ou de capitale, la banlieue dispute une certaine urbanite à la ville centrale et y participe à la fois. Cette évolution / révolution, basée sur les mobilités de travail, d'études, de loisirs, de consommation, fragmente l'espace urbain tout en y privilégiant et en y multipliant les espaces de propriété privée (complexes récréotouristiques, centres commerciaux, etc.).

L'étalement urbain, caractéristique maintenant inhérente au système territorial nord-américain, y compris au Québec, touche tant la capitale que la métropole. Il correspond aussi à l'ambivalence de la société quant à la valeur culturelle, sociale et morale de la densité urbaine et de la location comme statut $\mathrm{d}^{+}$occupation du logement. Dans ce contexte, la banlieue apparaît comme un compromis entre la ville et la campagne, favorisant l'accès quotidien à des loisirs privatisés et même en partie intégrés à l'espace domestique (piscine, cinéma-maison, etc.).

En tant que respectivement métropole et capitale, Montréal et Québec présentent des différences notables de taille, de fonctions urbaines, de caractéristiques architecturales dominantes dans leurs secteurs anciens et, surtout, d'écarts entre les espaces municipaux qui les composent. Les écarts sont en effet beaucoup plus prononcés dans la région métropolitaine de Montréal où l'internationalisation se concrétise par la présence active et visible de nombreux organismes internationaux et par l'intégration de communautés culturelles diversifiées et importantes développant des trajectoires résidentielles spécifiques. Malgré ces différences, Montréal et Québec représentent, à leurs façons respectives, la « nord-américanité urbaine s : étalement et spécialisation des quartiers résidentiés, dispersion du secteur tertiaire banalisé et des activités industrielles, prédominance des lois du marché sur les préceptes de la planification. On y trouve en quelque sorte un urbain complexe, éclaté, somme toute autant sinon plus suburbain qu'urbain, répondant au modèle du cycle de vie d'un produit où se développe done la dialectique déstructuration/restructuration, favorisant la reconversion des espaces anciens et leur mise en tourisme.

Si la pratique du tourisme urbain est fort ancienne, le discours sur cette forme spécifique de tourisme n'a émergé que récemment, au cours des dernières décennies. C'est que le tourisme urbain le plus traditionnel avait pour objet les villes mythi- ques, les villes-phares de nos civilisations ou des grands mouvements qui les ont marquées. Le tourisme urbain classique était dirigé par exemple vers Athènes, Rome, Jerusalem, Paris, Venise, Florence, etc. A ces destinations traditionnelles se sont ajoutées progressivement des destinations urbaines d'accueil de formes specifiques de tourisme : tourisme d'affaires à Londres, à New York, à Tokyo et dans d'autres métropoles ou mégalopoles du monde industrialisé ou post-industrialisé; tourisme sportif dans les villes d'accueil de Coupes du monde ou de Jeux olympiques; tourisme culturel aussi dans les villes les plus réputées pour leurs musées, leurs traditions des arts de la scène ou autres.

On observe, en parallèle au tourisme urbain classique et au tourisme sectoriel en milieu urbain qui prend forme dans les métropoles au cours des dernières décennies, un tourisme urbain de masse et en partie de proximité, ou de relative proximité, qui se caractérise par la consommation d'attractions, mais aussi du rythme de vie de la ville d'accueil, de son ambiance et de son animation fondée, entre autres, sur ses activités récréatives et sur les événements qui y sont organisés. La déambulation, particulièrement dans des espaces publics, constitue l'un des catalyseurs de l'expérience touristique du visiteur et de sa particularisation. C'est pourquoi la convivialité de la signalétique est si importante, de même que la qualité de l'accueil, la disponibilité de systèmes d'information basés sur des ressources humaines ou technologiques, la sécurité et l'animation.

Le tourisme de métropole au sens entendu ici résulte de la mise en tourisme de la ville 
même, de sa a spectarisation $\%$, de son émergence comme produit et attraction touristiques. Dans cette perspective, le tourisme métropolitain est examiné sous l'angle de l'offre s'adressant aux touristes au sens traditionnel du terme, ainsi qu'aux excursionnistes dont la distance parcourue est de 80 à 400 kilomètres. Cependant, la participation de la population locale est essentielle au succès de ce tourisme de métropole ; elle assure l'animation, particulièrement dans les espaces publics fréquentés par les touristes, ainsi qu'une partie de la valorisation et du succès des spectacles de masse qui encadrent les spectacles-performances d'élite offerts en salles, dans le cadre d'événements thématiques.

\section{Développement des fonctions touristiques et récréatives dans une conjoncture de crise urbaine}

C'est au moment où les métropoles sont particulièrement affectées par la restructuration imposée par la post-industrialisation que la fonction touristique prend un nouvel essor, se a spectacularise $x$ et envahit des espaces vonés à l'abandon. Le tourisme urbain participe à la réhabilitation des espaces centraux et bénéficie du paysage métropolitain, fut-il en ruines, lesquels peuvent devenir en eux-mêmes une attraction comme le démontre l'exemple convaincant du World Trade Center à New York. Les nouveaux amphitheâtres nord-américains les plus performants s installent aussi dans les espaces vacants des centres-villes et participent au mouvement de réanimation urbaine, dès leur mise en chantier, en devenant des attractions au titre même de leur érection.

Dans les transformations profondes et spectaculaires qui affectent le décor et l'espace urbains, le contenu et le contenant de la ville, mais aussi son atractivité et sa signification. le tourisme joue un rôle croissant qu'il devient injustifiable de négliger (Cazes et Polier, 1996: 87).

A Montréal, en particulier, les effets du tourisme sur la valorisation du centre-ville sont très marquếs à cause de la localisa= tion centrale du Palais des congrès et de la forte concentration territoriale du parc hôtelier.

La Ville de Montréal est aussi un lieu important de congres internationaux. Montreal se situe au moisieme rang parmi les villes nord-américaines a ce niveau, au premier rang au Canada (Conseil régional de développement de Thle de Montréal. Plan stratégique de développement 2000 . 2005, p. 32).
L'espace d'accueil des congrès et celui de la localisation des plus importants organismes à vocation internationale sont actuellement réunis aux fins de l'ếtablissement et de la reconnaissance officielle du Quartier international de Montréal (QIM).

\section{Le tourisme comme dérivatif à la ségrégation métropolitaine ef aux écarts de proximité}

La taille d'un marché métropolitain, la mobilité de sa population, la présence de

\section{L'Art Déco s'affiche...}

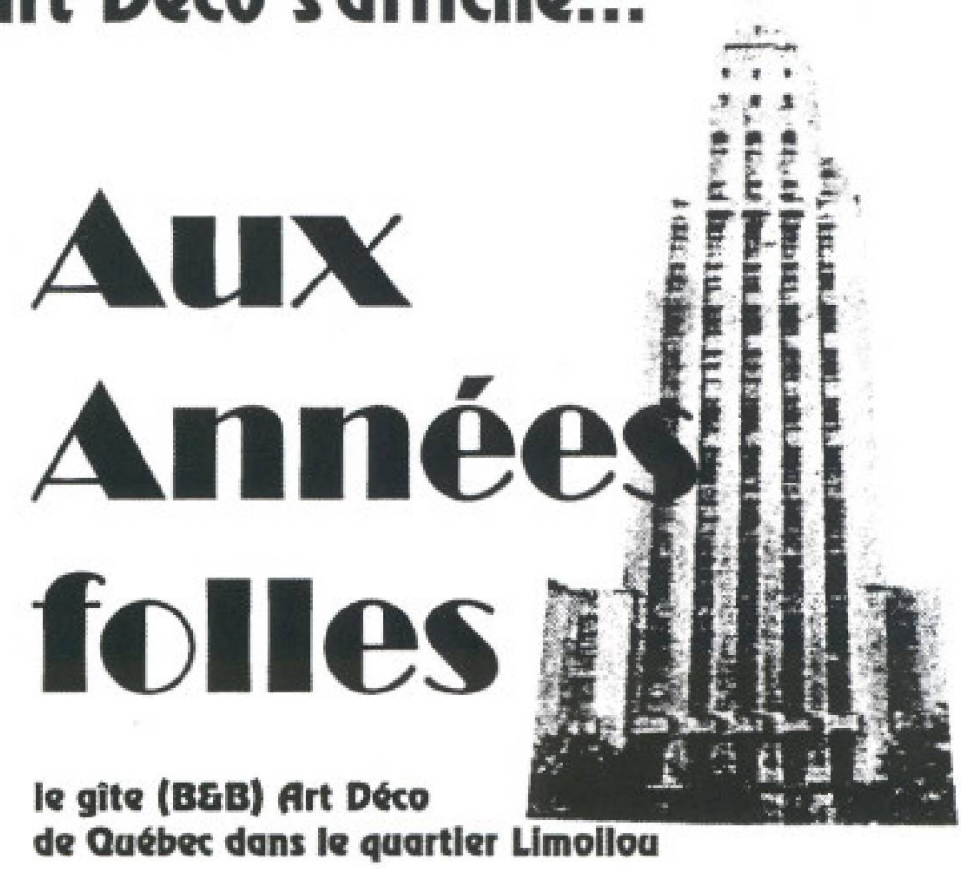

Une collection, an esprit, an lleu de sejoor d
découvili... Jean Dooust, prop. (418) $260-9549$

www.multimania.com/annfol 


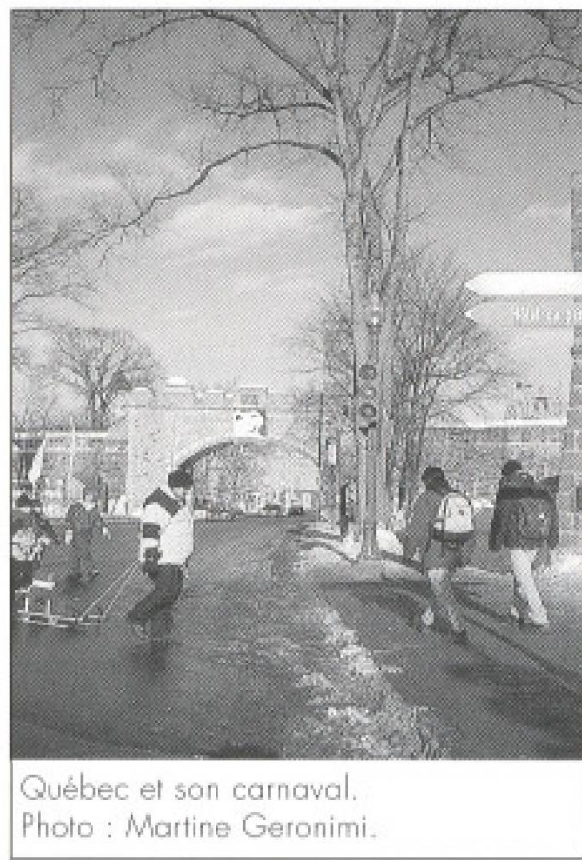

communautés culturelles en lien avec d'autres valeurs et d'autres marchés constituent, entre autres, des facteurs contributifs importants à l'expression d'écarts significatifs. En ce qui concerne la région métropolitaine de Montréal, ces écarts, souvent de proximité, ont été largement documentés (Pilette, 1996).

La région métropolitaine de Montréal se compose en effet de l'île centrale de Montréal et de sa couronne qui se partagent à peu près également une population totale de plus de 3,5 millions de personnes. Le modèle dominant est caractérisé par le choix de la propriété unifamiliale, généralement à l'extérieur de l'île, comme habitation de la jeune famille composée de deux parents et d'enfants au foyer. La proportion des groupes d'âge plus élevée est donc plus importante dans les quartiers centraux où s'observe parallèlement une féminisation significative. Les écarts de revenus des familles sont importants et reflètent la hiérarchisation des territoires résidentiels, «L'écart entre le revenu familial moyen du secteur le plus riche (Summit Circle à Westmount) et celui du secteur le plus pauve (Habitations Jeanne-Mance) est en fait dans un rapport de 13 à $I$ s (Drouilly, 1996 : 297).

Dans un tel contexte, les flux touristiques, avec l'intensité des echanges qu'ils sou- tiennent, apparaissent comme un liant, un antidote à la balkanisation intramétropolitaine.

En 2000, 5,8 millions de visiteurs ont séjourné plus de 24 heures à Montréal et leur's dépenses sont évaluées a 1,8 milliards de dollars (Tourisme Montréal, 2000 : 3 4), dont 1 milliard proviennent des touristes. internationaux.

Neuf événements montréalais ont obtenu des fréquentations respectives allant de 1600000 à 100000 personnes. Ce sont, dans l'ordre:

\section{Fréquentation des principaux événements Montréal $=2000$}

\begin{tabular}{|lr|}
\hline $\begin{array}{l}\text { Le Festival international } \\
\text { de Jarz de Montreal }\end{array}$ & 1600000 \\
\hline Le Festival Juste pour rire & 457000 \\
\hline $\begin{array}{l}\text { Mosileultures internationales } \\
\text { de Montreal }\end{array}$ & 731000 \\
\hline Les Francopholies & 600000 \\
\hline Diversicite & 600000 \\
\hline Le Grand Prix Players du Canadat & 272000 \\
\hline Les Internationaux du Cunada & 157236 \\
\hline Le Festival des Films du Monde & 350000 \\
\hline Festival Nuits d'Afrique & 100000 \\
\hline
\end{tabular}

Même si plusieurs de ces événements reposent d'abord sur les performances, en salles, d'artistes, de sportifs ou d'artisans d'élite, leur succès de fréquentations est aussi attribuable à leur portion de programmation qui comporte des activités populaires de masse, supportées par des espaces publics comme les rues et les places fermées, ẩ ces fins, â la circulation ou à l'accès automobile pendant des périodes limitées, donnant ainsi à la déambulation une valeur d'exception et même d'urgence qui ajoute et participe à son appréciation et à son caractère festif métropolitain.

Largement médiatisés, reposant en général sur de larges commandites du secteur privé, les grands événements montralais se tiennent dans une zone géographique centrale par rapport à l'espace métropolitain. Ils animent ces espaces et les occupent littéralement, relayant les fonctions tertiaires et d'affaires après les heures ouvrables et mểme coexistant, en partie, aux mêmes heures. Les grands événements participent donc à la fonction d'échange propre aux villes dont les métropoles sont caractérisées par l'intensité. Leur concentration dans des espaces centraux relève d'autant plus de la définition de la qualité de vie urbaine qu'elle constitue une opposition radicale à la dominante spécialisation des espaces en territoire métropolitain nord-américain.

Sans une activité touristique aussi intense, avec son nombre élevé de municipalités (101 avant les fusions de janvier 2002), la région métropolitaine de Montréal n'échapperait pas, en effet, au phénomène de ségrégation qui caractérise les espaces municipaux et à la spécialisation qui en résulte. Elle serait une métropole parmi d'autres. Si le tourisme urbain est largement un tourisme de l'expérience, de la consommation et de l'éphémère, il n'en permet pas moins une différenciation qui participe au rehaussement de la qualité de vie urbaine.

\section{Danielle Pilette est professeure titulaire} au Departement d'études urbaines et touristiques, à l'Ecole des sciences de la gestion de l'Université du Quebec à Montral.

\section{Bibliographie}

Cazes, Georges, et Françoise Potier (1996), Le tourisme urbain, Paris, Que sais-je, no 3191, p. 87 .

Conseil régional de développement de l'île de Montréal, Plan strategigue de developpement $2000-2005$, p. 32

Drouilly, Pierre (1996), Lespace social de Montreal, 195/-199/. Sillery, Septentrion, p. 297.

Pilette, Danielle (1996), \& Les spécificités locales et le découpage du territoire dans la région métropolitaine de Montréal w, dans Coopénation et concurrence, Montréal, Guérin.

Tourisme Montréal (2000), Bilan touristique 2000 , p. $3-4$. 